\title{
Planar dGEMRIC Maps May Aid Imaging Assessment of Cartilage Damage in Femoroacetabular Impingement
}

\author{
Evgeny Bulat MA, Sarah D. Bixby MD, Carl Siversson PhD, \\ Leslie A. Kalish ScD, Simon K. Warfield PhD, Young-Jo Kim MD, PhD
}

Published online: 25 August 2015

(C) The Association of Bone and Joint Surgeons (B) 2015

\begin{abstract}
Background Three-dimensional (3-D) delayed gadolinium-enhanced MRI of cartilage (dGEMRIC) helps quantify biochemical changes in articular cartilage that correlate with early-stage osteoarthritis. However, dGEMRIC
\end{abstract}

Each author certifies that he or she, or a member of his or her immediate family, has no funding or commercial associations (eg, consultancies, stock ownership, equity interest, patent/licensing arrangements, etc) that might pose a conflict of interest in connection with the submitted article.

All ICMJE Conflict of Interest Forms for authors and Clinical Orthopaedics and Related Research ${ }^{\mathbb{R}}$ editors and board members are on file with the publication and can be viewed on request.

Clinical Orthopaedics and Related Research ${ }^{\mathbb{R}}$ neither advocates nor endorses the use of any treatment, drug, or device. Readers are encouraged to always seek additional information, including FDA approval status, of any drug or device before clinical use. Each author certifies that his or her institution approved the human protocol for this investigation, that all investigations were conducted in conformity with ethical principles of research, and that informed consent for participation in the study was obtained.

This work was performed at Boston Children's Hospital, Boston, MA, USA.

\section{E. Bulat, Y.-J. Kim ( $\square)$}

Department of Orthopedic Surgery, Boston Children's Hospital, 300 Longwood Avenue, Boston, MA 02115, USA

e-mail: Young-Jo.Kim@childrens.harvard.edu

S. D. Bixby, S. K. Warfield

Department of Radiology, Boston Children's Hospital, Boston, MA, USA

C. Siversson

Department of Medical Radiation Physics, Lund University, Skåne University Hospital, Malmö, Sweden

L. A. Kalish

Clinical Research Center, Boston Children's Hospital, Boston, MA, USA analysis is performed slice by slice, limiting the potential of 3-D data to give an overall impression of cartilage biochemistry. We previously developed a computational algorithm to produce unfolded, or "planar," dGEMRIC maps of acetabular cartilage, but have neither assessed their application nor determined whether MRI-based grading of cartilage damage or dGEMRIC measurements predict intraoperative findings in hips with symptomatic femoroacetabular impingement (FAI).

Questions/purposes (1) Does imaging-based assessment of acetabular cartilage damage correlate with intraoperative findings in hips with symptomatic FAI? (2) Does the planar dGEMRIC map improve this correlation? (3) Does the planar map improve the correlation between the dGEMRIC index and MRI-based grading of cartilage damage in hips with symptomatic FAI? (4) Does the planar map improve imaging-based evaluation time for hips with symptomatic FAI?

Methods We retrospectively studied 47 hips of 45 patients with symptomatic FAI who underwent hip surgery between 2009 and 2013 and had a 1.5-T 3-D dGEMRIC scan within 6 months preoperatively. Our cohort included 25 males and 20 females with a mean \pm SD age at surgery of $29 \pm 11$ years. Planar dGEMRIC maps were generated from isotropic, sagittal oblique TrueFISP and T1 sequences. A pediatric musculoskeletal radiologist with experience in hip MRI evaluated studies using radially reformatted sequences. For six acetabular subregions (anterior-peripheral [AP]; anteriorcentral [AC]; superior-peripheral [SP]; superior-central [SC]; posterior-peripheral [PP]; posterior-central [PC]), modified Outerbridge cartilage damage grades were recorded and region-of-interest $\mathrm{T} 1$ averages (the dGEMRIC index) were measured. Beck's intraoperative cartilage damage grades were compared with the Outerbridge grades and dGEMRIC indices. For a subset of 26 hips, 13 were reevaluated with the 
map and 13 without the map, and total evaluation times were recorded.

Results There were no meaningful differences in the correlations obtained with versus without referencing the planar maps. Planar map-independent Outerbridge grades had a notable $(\mathrm{p}<0.05)$ Spearman's rank correlation $(\rho)$ with Beck's grades that was moderate in AP, SC, and PC $(0.3<\rho$ $<0.5)$ and strong in SP $(\rho>0.5)$. For map-dependent Outerbridge grades, $\rho$ was moderate in AP, AC, and SC and strong in SP. Map-independent dGEMRIC indices had a $\rho$ with Beck's grades that was moderate in AP and SC $(-0.3>$ $\rho>-0.5)$ and strong in SP $(\rho<-0.5)$. For map-dependent dGEMRIC indices, $\rho$ was moderate in SC and strong in SP. Similarly, there were no meaningful, map-dependent differences in the correlations. When comparing Outerbridge grades and dGEMRIC indices, there were notable correlations across all subregions. Without the planar map, $\rho$ was moderate in AC and PC and strong in AP, SP, SC, and PP. With the map, $\rho$ was strong in all six subregions. In $\mathrm{AC}$, there was a notable map-dependent improvement in this correlation $(\mathrm{p}<0.001)$. Finally, referencing the planar dGEMRIC map during evaluation was associated with a decrease in mean evaluation time, from $207 \pm 32$ seconds to $152 \pm 33$ seconds $(\mathrm{p}=0.001)$.

Conclusions Our work challenges the weak correlation between dGEMRIC and intraoperative findings of cartilage damage that was previously reported in hips with symptomatic FAI, suggesting that dGEMRIC has potential diagnostic use for this patient population. The planar dGEMRIC maps did not meaningfully alter the correlation of imaging-based evaluation of cartilage damage with intraoperative findings; however, they notably improved the correlation of dGEMRIC and MRI-based grading in AC, and their use incurred no additional time cost to imaging-based evaluation. Therefore, the planar maps may improve dGEMRIC's use as a continuous proxy for an otherwise discrete and simplified MRI-based grade of cartilage damage in hips with symptomatic FAI.

Level of Evidence Level III, diagnostic study.

\section{Introduction}

In a recent summary of the Global Burden of Disease 2010 Study [15], March and colleagues [15] highlighted that hip and knee osteoarthritis (OA) together account for $10.5 \%$ of global disability burden resulting from musculoskeletal (MSK) disorders. Furthermore, OA's global burden has increased by $65 \%$ since 1990 - more than any other MSK disorder category. Specifically in the hip, aberrant joint biomechanics resulting from conditions such as developmental hip dysplasia (DDH) and femoroacetabular impingement (FAI) can predispose individuals to early OA. Consequently, surgical techniques such as the periacetabular osteotomy (PAO), the safe surgical dislocation, and arthroscopic osteoplasty have been developed to remodel hip biomechanics, lessen the rate of OA progression, and extend the hip's natural lifetime [10].

Diagnostic MRI aids early-stage OA detection and assists in surgical decision-making. One technique, delayed gadolinium-enhanced MRI of cartilage (dGEMRIC), correlates with histological markers of early-stage OA [4] and pain symptoms associated with DDH and FAI $[11,13]$. In hips with DDH, dGEMRIC also correlates with the severity of dysplasia and can predict premature joint failure after treatment with PAO [8, 12, 13]. Briefly, glycosaminoglycans (GAG) constitute a major portion of the matrix in cartilage and hold a strongly negative charge. As a joint deteriorates, GAG is lost, thus reducing the overall negative charge of cartilage. In dGEMRIC, the negatively charged contrast agent gadopentetate dimeglumine (GdDPTA $^{2-}$ ) penetrates cartilage in relation to the loss of GAG. Because Gd-DPTA ${ }^{2-}$ reduces the $\mathrm{T} 1$ relaxation time, its increased presence in cartilage as measured by MRI quantifies biochemical degradation. Finally, because biochemical cartilage damage through loss of GAG content precedes radiographic signs of damage [21], dGEMRIC may aid the detection of early-stage OA.

Nonetheless, the evidence of MRI's ability to reliably predict cartilage damage in hips with symptomatic FAI has been mixed. Anderson et al [2] reported that preoperative MR arthrography has only $22 \%$ sensitivity for identifying cartilage delamination. On the other hand, Lattanzi et al [14] estimated dGEMRIC's sensitivity and specificity as $47 \%$ and $58 \%$, respectively. Furthermore, by using intrapatient signal standardization methods, the researchers could increase these values to over $70 \%$ and $60 \%$, respectively. Meanwhile, Bittersohl et al. [7] reported that dGEMRIC and intraoperative findings correlate poorly $(r=$ $0.114, \mathrm{p}<0.126$ ). However, we wondered whether there may still be meaningful, subregion-specific correlation between imaging-based (MRI or dGEMRIC) and intraoperative findings that would help validate MRI's use for detecting $\mathrm{OA}$ in the symptomatic FAI population.

Three-dimensional (3-D) dGEMRIC allows for quantitative assessment of changes in hip cartilage biochemistry [13]; however, the image slices that are generated are twodimensional and challenging to interpret clinically. Our group developed a way to automatically process $1.5-\mathrm{T}$ 3-D dGEMRIC data to create a flattened image of the hip cartilage "horseshoe" that is analogous to a map of the earth's surface [19]. Briefly, the algorithm (1) takes an isotropic morphology sequence (TrueFISP), two isotropic T1 sequences (3-D variable flip angle or 3-D VFA), and a dGEMRIC sequence as inputs; (2) performs automated segmentation of the morphology data into femur, cartilage, and acetabulum using a reference set of manually 
segmented studies; (3) applies the generated segmentation field to the dGEMRIC data; (4) isolates and radially averages the acetabular portion of the cartilage segment; and (5) unfolds the result into a planar, equal-area projection. This method therefore permits the visual assessment of local biochemical changes in any acetabular subregion (Fig. 1).

Although this algorithm appeared promising, we did not know if it would be useful clinically. We were therefore interested in seeing whether the map derived from our algorithm correlated with surgical findings. Meanwhile, because testing the planar map's utility would entail using intraoperative findings as a standard of comparison, we were interested in reexamining the consistency of both MRI-based grades and dGEMRIC with intraoperative findings across specific subregions of acetabular cartilage (Fig. 1B).

We therefore asked: (1) Does imaging-based assessment of acetabular cartilage damage correlate with intraoperative findings in hips with symptomatic FAI? (2) Does the planar dGEMRIC map improve this correlation? (3) Does the planar map improve the correlation between the dGEMRIC index and MRI-based grading of cartilage damage in hips with symptomatic FAI? (4) Does the planar map improve imaging-based evaluation time for hips with symptomatic FAI?

\section{Patients and Methods}

This was a retrospective study approved by our institutional review board. The study cohort consisted of 47 hips in 45 patients who underwent a 3-D dGEMRIC scan as part of a diagnostic workup for FAI between September 2008 and April 2013 and subsequently had FAI-correcting surgery.

Patients were included in the study if they (1) had a diagnosis of symptomatic FAI; (2) underwent surgery to treat the FAI; (3) underwent a 1.5-T 3-D dGEMRIC scan within 6 months preoperatively that included both TrueFISP and dGEMRIC sequences; and (4) were part of our institutional review board-approved surgical hip registry. Patients were excluded if the dGEMRIC scan was deemed of nondiagnostic quality or if the original record of intraoperative findings was absent.

Planar dGEMRIC maps were generated from isotropic sagittal oblique TrueFISP and T1 mapping sequences using core computing clusters at the Boston Children's Computational Radiology Laboratory (www.crl.med.harvard.edu) as well as Harvard Medical School's Orchestra cluster (https://rc.hms.harvard.edu/\#orchestra). The lead author (EB) applied the segmentation algorithm and, in consultation with the algorithm's developer (CS), checked the quality of each record's segmentation using the visualization program, ITK-SNAP [22] (www.itksnap.org). For those records that demonstrated satisfactory overlay between the $\mathrm{T} 1$ segmentation and the morphology sequence, a pediatric musculoskeletal radiologist with experience in hip MRI (SDB) performed all subsequent assessment on deidentified studies.

Radial reformatting of each study was performed as follows, in a manner that is generally consistent with previously published methodology [9]. The sagittal oblique TrueFISP and DFE VIBE T1 (dGEMRIC) sequence s were reformatted into radially oriented plane sequences using the femoral neck as a standard axis of rotation (Fig. 2A-B). Reformatting was performed using Synapse 3D software (Fujifilm Corp, Tokyo, Japan). Eighteen radially reformatted TrueFISP images were acquired at $0.63 \mathrm{~mm}$ thickness at $10^{\circ}$ intervals, and 18 radially reformatted dGEMRIC images were acquired at $2 \mathrm{~mm}$ thickness at $10^{\circ}$ intervals. The inferior radial plane was defined by the image in which the transverse ligament is visualized in the absence of both the anterior and posterior bony acetabulum (ie, the inferior-most aspect of the acetabular "horseshoe"shaped articular surface). The superior (S) sector was defined by the radial plane bisecting the inferior joint and the four radial planes anterior and posterior to this plane (a sector spanning $80^{\circ}$ of joint space). The anterior (A) sector was defined by the radial plane rotated $90^{\circ}$ in a clockwise (right hip) or counterclockwise (left hip) direction from the inferior plane and included the four radial imaging planes superior and inferior to this plane. The posterior $(\mathrm{P})$ sector was defined by rotating the radial plane $90^{\circ}$ in the opposite direction from the inferior plane and included the four radial imaging planes superior and inferior to this plane. Reformatted images were saved as DICOM images and sent to a PACS workstation for review.

For each of the sectors (A, S, P), the image slice containing the morphologically or biochemically worstlooking acetabular cartilage for the peripheral half $(\mathrm{P}$, bound by the acetabular rim) was chosen, and another one was chosen for the central half $(\mathrm{C}$, bound by the acetabular fossa). This division scheme yielded six acetabular subregions: AP, AC, SP, SC, PP, and PC. For each subregion, cartilage damage was assessed on the morphology sequence (Fig. 2C) using the modified Outerbridge grade (Table 1), a standard cartilage damage rating scale (referred to simply as the "Outerbridge grade"). Using the corresponding dGEMRIC sequence (Fig. 2D), the mean T1 value from a region-of-interest trace within the acetabular cartilage (the dGEMRIC index) was also measured for each subregion.

Through this approach, one of the authors (SDB) obtained two sets of imaging-based assessments per study from radial reformats at least 3 weeks apart: one without 


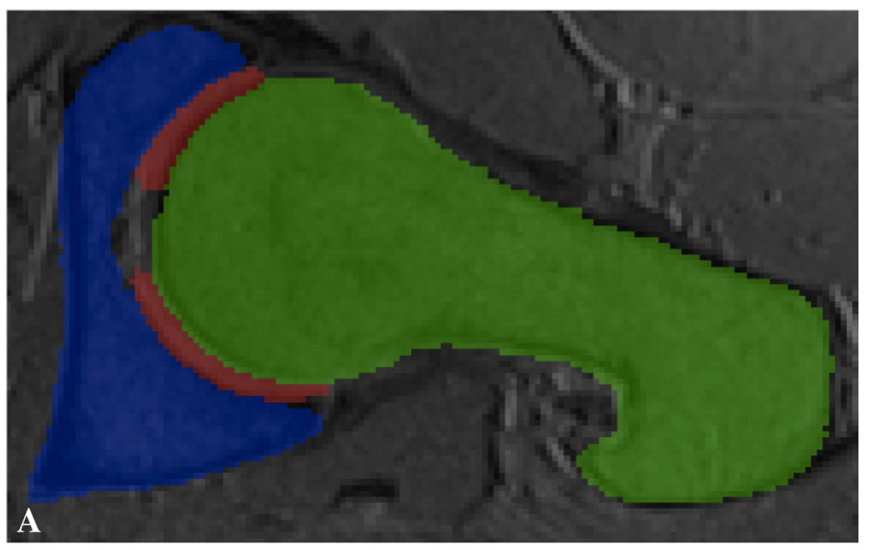

\section{Acetabular Subregions}
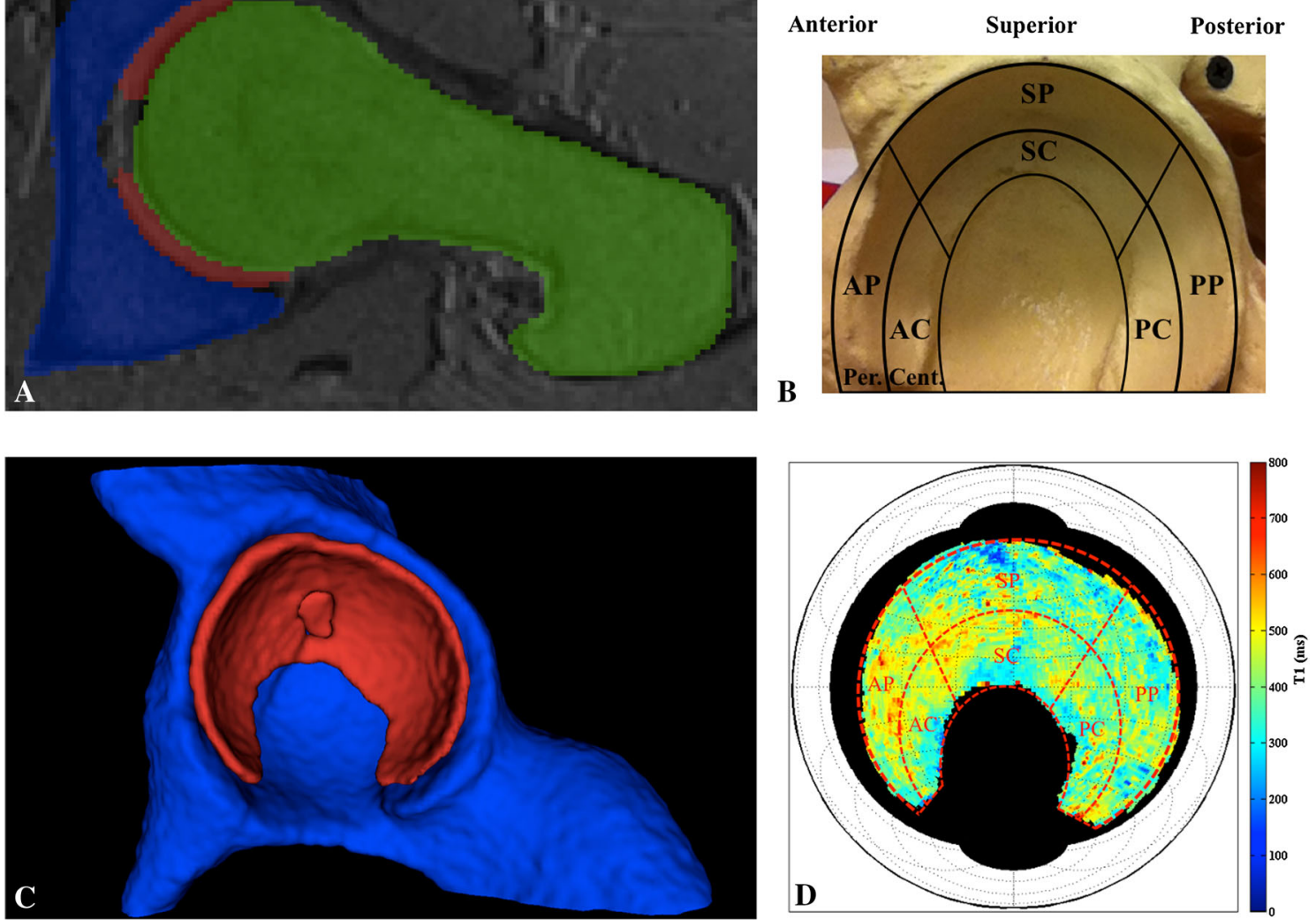

Fig. 1A-D (A) The planar dGEMRIC map algorithm [19] takes 3-D, isotropic, 1.5-T dGEMRIC hip sequences and performs automated segmentation of the data to generate the acetabular (blue), cartilage (red), and femoral (green) segments. Shown is a sagittal oblique view of a $\mathrm{T} 1$ sequence slice with the segmentation overlay, visualized in ITK-SNAP [22]. (B) The defined acetabular subregions are overlaid on top of a pelvic model for reference. $\mathrm{AP}=$ anterior-peripheral; $\mathrm{AC}=$ anterior-central; $\mathrm{SP}=$ superior-peripheral; $\mathrm{SC}=$ superior-central; $\mathrm{PP}=$ posterior-peripheral; $\mathrm{PC}=$ posterior-central. $(\mathbf{C})$ The cartilage

visual reference to the corresponding planar dGEMRIC map and another with visual reference to the map. The map was projected on a separate screen and available for reference during evaluation but was not directly used to derive measurements. Instead, the author (SDB) was instructed to refer to the map when choosing the study imaging planes with cartilage damage. To lessen potential order bias, 19 hips were randomly selected to be the first evaluated with the planar map's assistance and then without. All other hips were evaluated in reverse order.

At least 4 weeks after evaluating the entire hip cohort, the author (SDB) reevaluated a subset of 26 hips by Outerbridge grade and the dGEMRIC index for the purpose of calculating intrarater reliability. Thirteen of these hips were reevaluated without the use of a planar dGEMRIC map, and 13 were reevaluated with the use of a planar map. For all 26 hips, the time it took to perform the evaluation was recorded. segment (red) is shown, from which the data are extracted and further processed (visualized in ITK-SNAP). Behind it is the acetabular segment (blue), whereas the femoral segment is not shown. (D) Radial averaging and unfolding of the cartilage data yield a planar map that displays the acetabular dGEMRIC signal (visualized in MATLAB, www.mathworks.com). The red, dashed subregion grid from $B$ is overlaid on top of the planar map for clarifying purposes only.

For each acetabular subregion, the orthopaedic surgeon assigned an intraoperative grade using the Beck scale (Table 1) at the time of surgery. These data, collectively originating from three orthopaedic surgeons, composed the intraoperative standard.

The variables analyzed included the Beck's grade, the Outerbridge grade, the dGEMRIC index in milliseconds, and radiological evaluation time in seconds. For the Beck's grade, the average weighted Cohen's kappas $(\kappa)$ for intraand interrater reliability were previously reported as 0.80 and 0.65 , respectively [17]. For the Outerbridge grade, $\kappa$ for the author's (SDB) intrarater reliability was calculated as 0.64 across all acetabular subregions. For the dGEMRIC index, the intraclass correlation for the author's (SDB) intrarater reliability was 0.89 across all subregions.

All statistical analysis was performed by two of the authors (EB, LAK) using MATLAB (MathWorks, Inc, Natick, MA, USA) and R (www.r-project.org) [18]. 

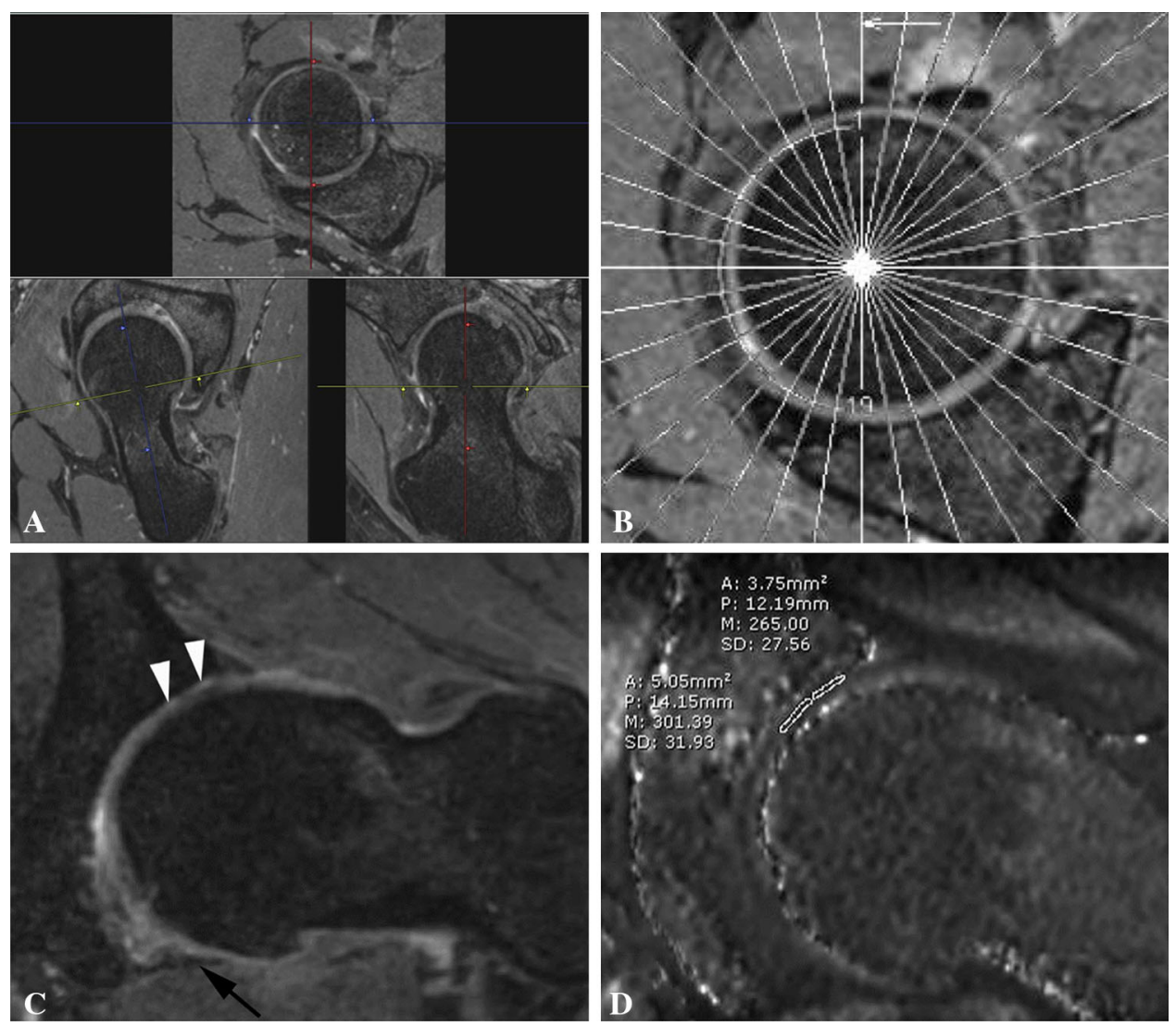

Fig. 2A-D (A) The figure shows the reformatting technique for sagittal oblique TrueFISP sequence. Planes are aligned along the femoral neck axis and rotate around a fixed central point in the femoral neck. (B) A localizer image demonstrating the axis of rotation of the radially reformatted TrueFISP sequence is shown. Image planes rotate around a fixed central point in the femoral neck. (C) The figure shows a reformatted radial TrueFISP image

demonstrating areas of full-thickness acetabular cartilage loss (white arrowheads). The transverse ligament is denoted by the black arrow. (D) The figure shows a reformatted radial DFE VIBE TI map (dGEMRIC) image demonstrating the measurement of the dGEMRIC index in the superior acetabular cartilage with indices of $301 \mathrm{msec}$ centrally and $265 \mathrm{msec}$ peripherally.

Table 1. An overview of the MRI-based modified Outerbridge grade [3] and the intraoperative Beck's grade [5]

\begin{tabular}{|c|c|}
\hline Modified Outerbridge grade & Beck's grade \\
\hline $0=$ Normal cartilage & $1=$ Normal \\
\hline $\begin{array}{l}1 \text { = Signal changes and/or } \\
\text { minor surface irregularities }\end{array}$ & $\begin{array}{r}2=\text { Malacia (roughening } \\
\text { of surface, fibrillation) }\end{array}$ \\
\hline $\begin{array}{l}2=\text { Cartilage thickness } \\
\text { loss } \leq 50 \%\end{array}$ & $\begin{array}{l}3 \text { = Debonding (loss of fixation } \\
\text { to the subchondral } \\
\text { bone; carpet phenomenon) }\end{array}$ \\
\hline $\begin{array}{l}3=\text { Cartilage thickness } \\
\text { loss }>50 \%\end{array}$ & $\begin{array}{l}4 \text { = Cleavage (loss of fixation } \\
\text { to the subchondral bone; } \\
\text { frayed edges, thinning of } \\
\text { the cartilage flap) }\end{array}$ \\
\hline $4=$ Total cartilage loss & $\begin{array}{l}5=\text { Full-thickness defect, complete } \\
\text { loss of cartilage }\end{array}$ \\
\hline
\end{tabular}

calculated separately from planar dGEMRIC map-dependent data and planar map-independent data, and 95\% confidence intervals and $\mathrm{p}$ values were presented for each using the Fisher Z-transformation approach. We tested whether the correlations with versus without the use of the planar maps differed from each other using methods in either Meng et al. [16] or Steiger [20] depending on whether the pairs of correlated measures being compared had one measure in common. When comparing the planar map-dependent and map-independent Beck-Outerbridge correlations as well as map-dependent and map-independent Beck-dGEMRIC correlations, the same Beck's grades are used in every correlation and so the methods of Meng et al. were used. In the Outerbridge-dGEMRIC analysis, both Outerbridge and dGEMRIC data were measured with and without the maps and thus the methods of Steiger were used. Finally, the Wilcoxon rank-sum test was used to nonparametrically test the significance of difference in planar map-dependent and map-independent radiological

Spearman's rank correlation was used to measure the pairwise correlations among Beck's grade, the Outerbridge grade, and the dGEMRIC index. The correlations were 
evaluation times. Two-sided $\mathrm{p}$ values were used for all comparisons, and the statistical significance threshold $\alpha$ was set to 0.05 .

The study cohort comprised 47 hips from 45 patients. Twenty-five patients were male and 20 were female. The distribution of the patients' age at surgery had a mean \pm SD of $29 \pm 11$ years and ranged from 14 years to 57 years. Forty-four hips had a maximum radial alpha $(\alpha)$ angle on the radial reformat greater than or equal to $60^{\circ}$, which is a previously proposed cutoff for normal morphology [1]. Five hips had a lateral center-edge angle of Wiberg greater than or equal to $40^{\circ}$, which we used as the upper cutoff for normal morphology. Four hips had a minimum joint space width less than or equal to $2.5 \mathrm{~mm}$ as measured on the AP view. Given the definitions of cam FAI $(\alpha \geq 60)$ and pincer FAI (lateral center-edge angle of Wiberg $\geq 40$ ), 39 hips had exclusively cam FAI, none had exclusively pincer FAI, five had combined (cam + pincer) FAI, and three had no radiographic signs of FAI despite impingement symptoms (Table 2). To clarify, although the crossover sign is sometimes also considered for the diagnosis of pincer FAI, it is quite sensitive to the improper projection of the pelvis in the AP radiograph view. Consequently, we excluded it from consideration.

Regarding presurgical MRI, patients underwent a dGEMRIC scan an average of $2.3 \pm 1.5$ months before surgery and ranging from 1 day to 6 months.

From our surgical hip registry, we identified 68 hips that underwent surgery between January 2009 and December

Table 2. Study cohort characteristics

\begin{tabular}{ll}
\hline & Values \\
\hline Patient demographics & \\
Total hips & 47 \\
Total patients & 45 \\
Male sex & $25(56 \%)$ \\
Age at surgery (years; mean \pm SD) & $29 \pm 11$ \\
Age range (years) & $14-57$ \\
Time between MRI and surgery (months; mean \pm SD) & $2.3 \pm 1.5$ \\
Time range (months) & $0.03-5.9$ \\
Radiographic description & \\
Minimum joint space width $\leq 2.5$ mm & $4(9 \%)$ \\
Lateral center-edge angle $\geq 40^{\circ}$ (pincer) & $5(11 \%)$ \\
Maximum $\alpha$ angle $\geq 60^{\circ}($ cam) & $44(94 \%)$ \\
FAI description & \\
Cam FAI only & $39(83 \%)$ \\
Pincer FAI only & $0(0 \%)$ \\
Combined (cam + pincer) FAI & $5(11 \%)$ \\
No radiographic signs of FAI & $3(6 \%)$ \\
\hline
\end{tabular}

FAI = femoroacetabular impingement.
2013 (inclusive) and also had a 3-D 1.5-T dGEMRIC scan within 6 months preoperatively. The scan included the following isotropic sequences necessary for processing through our algorithm: (1) a TrueFISP volume acquired at $256 \times 256 \times 144$ voxel density using a $(0.63 \mathrm{~mm})^{3}$ voxel resolution; (2) a dGEMRIC T1 volume acquired at $384 \mathrm{x}$ 384 x 96 voxel density using a $(0.83 \mathrm{~mm})^{3}$ voxel resolution. Thirteen of 68 hips had study sequence alignment issues that led to automated segmentation errors, and another eight lacked intraoperative records. This left 47 hips for the study. Later in the analysis stage, it was discovered that the Beck's intraoperative grades for the acetabular cartilage of three of the 47 hips were not recorded during surgery; as a result, we excluded these hips from analysis involving Beck's grade but retained them for all other analyses.

\section{Results}

Imaging-based assessment of acetabular cartilage damage by MRI morphology (through Outerbridge grade) or biochemistry (through the dGEMRIC index) correlates moderately to strongly with intraoperative findings in important acetabular subregions of hips with FAI. Regardless of whether or not the planar dGEMRIC map was used to aid evaluation, subregion-specific correlations were noted between the Outerbridge and Beck's grades (Table 3) as well as between the dGEMRIC index and Beck's grade (Table 4). In the case of Outerbridge grade versus Beck's grade, correlation was moderate in AP and SC $(0.3<\rho<0.5)$ and strong in SP $(\rho>0.5)$; likewise, for the dGEMRIC index versus Beck's grade, correlation was moderate in AP and SC $(-0.3>\rho>-0.5)$ and strong in SP $(\rho<-0.5)$. Importantly, the Outerbridge grade and the dGEMRIC index both correlated well with Beck's grade in AP and SP, the two subregions that demonstrated the widest range of cartilage damage severity (data not shown) and are most commonly damaged in hips with FAI [5] (Fig. 3A-B).

Although imaging-based and intraoperative findings did correlate, we saw no notable, planar map-dependent effects on the strength of their correlation across the acetabular subregions. For Outerbridge grade versus Beck's grade, none of the subregion-specific $p$ values for the comparison of map-dependent and map-independent correlation coefficients, $p_{\text {diff }}$, were below the significance cutoff $(p<0.05)$ (Table 3). For the dGEMRIC index versus Beck's grade, only the $\mathrm{p}_{\mathrm{diff}}$ for PC was low enough to potentially reject the null hypothesis of no map-dependent effect; however, both correlations were quite small $(-0.2<\rho<0.2)$. An analysis of the dGEMRIC index's ability to classify normal and damaged cartilage, ie, to distinguish Beck's grade of 
Table 3. Spearman's rank correlations and $95 \%$ confidence intervals for the modified Outerbridge grade versus Beck's intraoperative grade

\begin{tabular}{|c|c|c|c|}
\hline $\begin{array}{l}\text { Acetabular cartilage } \\
\text { subregion }\end{array}$ & No map & With map & $\begin{array}{l}\text { No map versus with } \\
\text { map difference }\end{array}$ \\
\hline \multirow[t]{2}{*}{ AP } & $0.40(0.12-0.62)$ & $0.39(0.10-0.61)$ & \multirow[t]{2}{*}{$\mathrm{p}_{\text {diff }}=0.94$} \\
\hline & $\mathrm{p}=0.008$ & $\mathrm{p}=0.009$ & \\
\hline \multirow[t]{2}{*}{$\mathrm{AC}$} & $0.14(-0.17$ to 0.42$)$ & $0.34(0.05-0.58)$ & \multirow[t]{2}{*}{$\mathrm{p}_{\text {diff }}=0.15$} \\
\hline & $\mathrm{p}=0.38$ & $\mathrm{P}=0.02$ & \\
\hline \multirow[t]{2}{*}{ SP } & $0.53(0.28-0.71)$ & $0.52(0.27-0.71)$ & \multirow[t]{2}{*}{$\mathrm{p}_{\text {diff }}=0.96$} \\
\hline & $\mathrm{p}<0.001$ & $\mathrm{p}<0.001$ & \\
\hline \multirow[t]{2}{*}{$\mathrm{SC}$} & $0.41(0.13-0.63)$ & $0.42(0.14-0.64)$ & \multirow[t]{2}{*}{$\mathrm{p}_{\text {diff }}=0.93$} \\
\hline & $\mathrm{p}=0.006$ & $\mathrm{p}=0.005$ & \\
\hline \multirow[t]{2}{*}{ PP } & $0.12(-0.19$ to 0.40$)$ & $0.16(-0.15$ to 0.44$)$ & \multirow[t]{2}{*}{$\mathrm{p}_{\text {diff }}=0.75$} \\
\hline & $\mathrm{p}=0.46$ & $\mathrm{P}=0.30$ & \\
\hline \multirow[t]{2}{*}{ PC } & $0.37(0.08-0.061)$ & $0.28(-0.03$ to 0.53$)$ & \multirow[t]{2}{*}{$\mathrm{p}_{\text {diff }}=0.49$} \\
\hline & $\mathrm{p}=0.01$ & $\mathrm{p}=0.07$ & \\
\hline
\end{tabular}

$\mathrm{AP}=$ anterior-peripheral; $\mathrm{AC}=$ anterior-central; $\mathrm{SP}=$ superior-peripheral; $\mathrm{SC}=$ superior-central; $\mathrm{PP}=$ posterior-peripheral; $\mathrm{PC}=$ posteriorcentral.

Table 4. Spearman's rank correlations and $95 \%$ confidence intervals for the dGEMRIC index versus Beck's intraoperative grade

\begin{tabular}{|c|c|c|c|}
\hline $\begin{array}{l}\text { Acetabular cartilage } \\
\text { subregion }\end{array}$ & No map & With map & $\begin{array}{l}\text { No map versus with } \\
\text { map difference }\end{array}$ \\
\hline $\mathrm{AP}$ & $\begin{array}{l}-0.35(-0.58 \text { to }-0.06) \\
p=0.02\end{array}$ & $\begin{array}{l}-0.27(-0.52 \text { to } 0.04) \\
p=0.08\end{array}$ & $\mathrm{p}_{\mathrm{diff}}=0.56$ \\
\hline $\mathrm{AC}$ & $\begin{array}{l}-0.03(-0.32 \text { to } 0.27) \\
p=0.87\end{array}$ & $\begin{array}{l}-0.21(-0.47 \text { to } 0.10) \\
p=0.18\end{array}$ & $\mathrm{p}_{\mathrm{diff}}=0.22$ \\
\hline SP & $\begin{array}{l}-0.63(-0.78 \text { to }-0.41) \\
p<0.001\end{array}$ & $\begin{array}{l}-0.57(-0.74 \text { to }-0.33) \\
\mathrm{p}<0.001\end{array}$ & $\mathrm{p}_{\mathrm{diff}}=0.59$ \\
\hline SC & $\begin{array}{l}-0.32(-0.56 \text { to }-0.02) \\
p=0.04\end{array}$ & $\begin{array}{l}-0.31(-0.56 \text { to }-0.01) \\
p=0.04\end{array}$ & $\mathrm{p}_{\mathrm{diff}}=0.96$ \\
\hline PP & $\begin{array}{l}-0.04(-0.34 \text { to } 0.26) \\
p=0.80\end{array}$ & $\begin{array}{l}-0.08(-0.37 \text { to } 0.23) \\
p=0.61\end{array}$ & $\mathrm{p}_{\mathrm{diff}}=0.79$ \\
\hline $\mathrm{PC}$ & $\begin{array}{l}-0.17(-0.45 \text { to } 0.13) \\
p=0.26\end{array}$ & $\begin{array}{l}0.14(-0.17 \text { to } 0.42) \\
p=0.38\end{array}$ & $\mathrm{p}_{\mathrm{diff}}=0.04$ \\
\hline
\end{tabular}

dGEMRIC = delayed gadolinium-enhanced MRI of cartilage; $\mathrm{AP}=$ anterior-peripheral; $\mathrm{AC}=$ anterior-central; $\mathrm{SP}=$ superior-peripheral; $\mathrm{SC}=$ superior-central; PP = posterior-peripheral; $\mathrm{PC}=$ posterior-central.

1 versus greater than 1 in either AP or SP, yielded sensitivities and specificities that are consistent with those previously reported but indicated no meaningful, map-dependent effect (data not shown). On the whole, planar dGEMRIC maps appear to neither increase nor decrease the consistency of MRI-based grading and dGEMRIC index with intraoperative findings.

The Outerbridge grade and the dGEMRIC index correlate well with each other across all acetabular subregions (Table 5). Without the planar map, correlation is moderate in AC and PC $(-0.3>\rho>-0.5)$ and strong in AP, SP, SC, and $\mathrm{PP}(\rho<-0.5)$. With the map, correlation is strong in all subregions. Like before, we highlight that, in the more damaged subregions AP and SP, strong correlations were noted (Fig. 3C). When testing for a planar map-dependent effect on the correlation between the Outerbridge grade and the dGEMRIC index, AC witnessed a marked improvement in $\rho$ from -0.41 to -0.79 ( $\left.p_{\text {diff }}<0.001\right)$. This translated to a notably smoother and more predictable drop in the dGEMRIC index with an increase in Outerbridge grade (Fig. 4). In all other subregions, $p_{\text {diff }}$ was below the threshold of significance.

Finally, our analysis of total imaging-based evaluation times with and without the help of the planar map suggests that the map increases evaluation speed. Although planar map-independent evaluation times had a mean \pm SD of $207 \pm 32$ seconds, planar map-dependent times had a mean $\pm \mathrm{SD}$ of $152 \pm 33$ seconds (Fig. 5). Therefore, the planar 

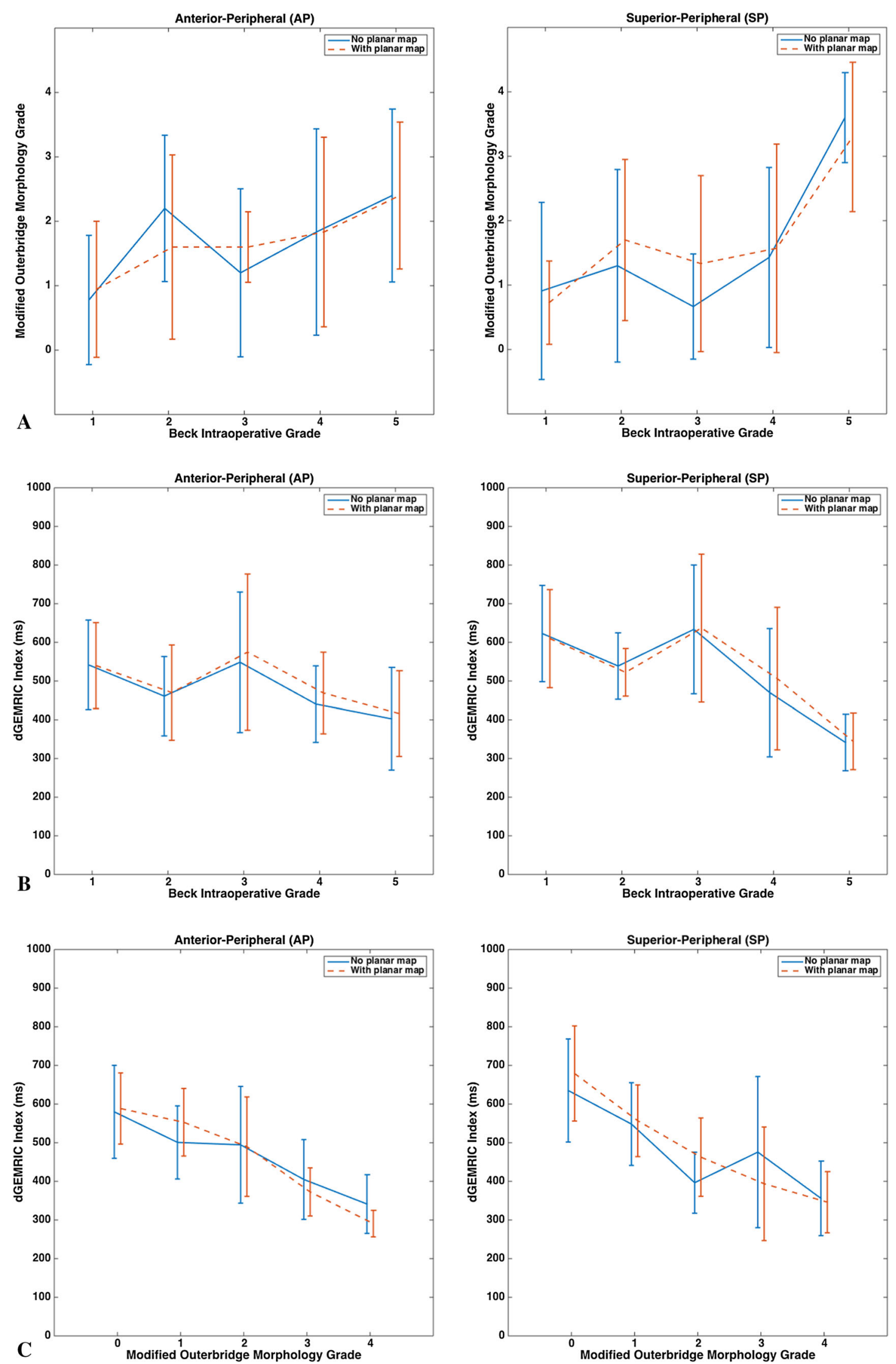
4Fig. 3A-C The figure shows the plots comparing radiological and surgical assessments of cartilage damage for acetabular subregions AP (left column) and SP (right column), which face the highest likelihood of damage in hips with FAI [5]. Planar dGEMRIC mapdependent and map-independent radiological assessment data are displayed side by side in each plot. The mean values are connected by either solid or dashed lines and the error bars indicate \pm SD. (A) The figure shows the modified Outerbridge grade (radiological) versus Beck's grade (surgical); (B) the dGEMRIC index (radiological) versus Beck's grade (surgical); (C) the dGEMRIC index (radiological) versus the modified Outerbridge grade (radiological), examining intermetric consistency in radiological assessment.

map makes evaluation 55 seconds, or $27 \%$, faster on average $(\mathrm{p}=0.001)$.

\section{Discussion}

dGEMRIC has been shown to quantify the biochemical changes that are associated with early-stage OA; however, the current mode of clinical evaluation with dGEMRIC limits one's ability to get a big picture view of hip articular cartilage damage. In addition, an analysis of how well dGEMRIC and other MRI-based findings correlate with intraoperative findings of cartilage damage in hips with symptomatic FAI has been limited. In this study, we applied a previously developed algorithm for generating planar maps of acetabular dGEMRIC values to MRI scans of surgical hips with symptomatic FAI. We measured how well both the MRI-based Outerbridge grading of cartilage morphology and the dGEMRIC index correlate with intraoperative findings and then tested what effect our planar maps have on that correlation. Our findings suggest that both the Outerbridge grade and the dGEMRIC index correlate moderately to strongly with Beck's intraoperative grade in acetabular subregions that most commonly suffer cartilage damage in hips with FAI. Meanwhile, the planar dGEMRIC maps may improve the speed of radiological evaluation by as much as $27 \%$ without affecting this correlation.

This study had a number of limitations. First, the study cohort comprised only 47 hips (44 hips for comparison to intraoperative findings). This modest number could account for some of the challenges encountered in capturing planar dGEMRIC map-dependent effects on the radiologist's assessment. It is worth noting, however, that the cohort was demographically diverse with respect to age and sex, indicating that the use of the maps may be widely applicable. Second, because the study was retrospective in design, and three different surgeons performed the surgeries, this may lead to variability in the intraoperative grading of cartilage damage. Therefore, the moderate-tostrong correlation between imaging-based and intraoperative assessments of cartilage damage despite this limitation suggests even stronger potential correlations. Our study was not designed to address correlation with clinical findings such as pain or motion, and we thus cannot speak to outcomes or prognosis. Lastly, the practical limitation of incorporating the planar map into a clinical workflow made it challenging to ascertain its potential clinical use. Future work toward optimizing the automated processing of dGEMRIC data such that the planar maps may be introduced into a clinical workflow at the time of initial interpretation will be helpful in this regard.

Our data show that MRI-based cartilage morphology grading and the dGEMRIC index both correlate moderately $(0.3<|\rho|<0.5)$ to strongly $(|\rho|>0.5)$ with intraoperative findings of cartilage damage in subregions AP, SP, and SC

Table 5. Spearman's rank correlations and $95 \%$ confidence intervals for the modified Outerbridge grade versus the dGEMRIC index

\begin{tabular}{|c|c|c|c|}
\hline $\begin{array}{l}\text { Acetabular cartilage } \\
\text { subregion }\end{array}$ & No map & With map & $\begin{array}{l}\text { No map versus } \\
\text { with map difference }\end{array}$ \\
\hline $\mathrm{AP}$ & $\begin{array}{l}-0.55(-0.72 \text { to }-0.31) \\
\mathrm{p}<0.001\end{array}$ & $\begin{array}{l}-0.71(-0.83 \text { to }-0.53) \\
\mathrm{p}<0.001\end{array}$ & $\mathrm{p}_{\mathrm{diff}}=0.12$ \\
\hline $\mathrm{AC}$ & $\begin{array}{l}-0.41(-0.62 \text { to }-0.14) \\
p=0.005\end{array}$ & $\begin{array}{l}-0.79(-0.88 \text { to }-0.65) \\
\mathrm{p}<0.001\end{array}$ & $\mathrm{p}_{\mathrm{diff}}<0.001$ \\
\hline SP & $\begin{array}{l}-0.71(-0.83 \text { to }-0.53) \\
\mathrm{p}<0.001\end{array}$ & $\begin{array}{l}-0.77(-0.87 \text { to }-0.62) \\
\mathrm{p}<0.001\end{array}$ & $\mathrm{p}_{\mathrm{diff}}=0.39$ \\
\hline $\mathrm{SC}$ & $\begin{array}{l}-0.75(-0.85 \text { to }-0.58) \\
\mathrm{p}<0.001\end{array}$ & $\begin{array}{l}-0.71(-0.83 \text { to }-0.53) \\
\mathrm{p}<0.001\end{array}$ & $\mathrm{p}_{\mathrm{diff}}=0.59$ \\
\hline $\mathrm{PP}$ & $\begin{array}{l}-0.58(-0.74 \text { to }-0.35) \\
p<0.001\end{array}$ & $\begin{array}{l}-0.51(-0.69 \text { to }-0.26) \\
\mathrm{p}<0.001\end{array}$ & $\mathrm{p}_{\mathrm{diff}}=0.58$ \\
\hline $\mathrm{PC}$ & $\begin{array}{l}-0.32(-0.56 \text { to }-0.04) \\
\mathrm{p}=.03\end{array}$ & $\begin{array}{l}-0.52(-0.70 \text { to }-0.27) \\
p<0.001\end{array}$ & $\mathrm{p}_{\mathrm{diff}}=0.24$ \\
\hline
\end{tabular}

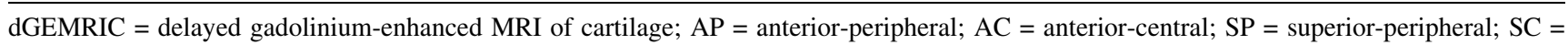
superior-central; $\mathrm{PP}=$ posterior-peripheral; $\mathrm{PC}=$ posterior-central. 


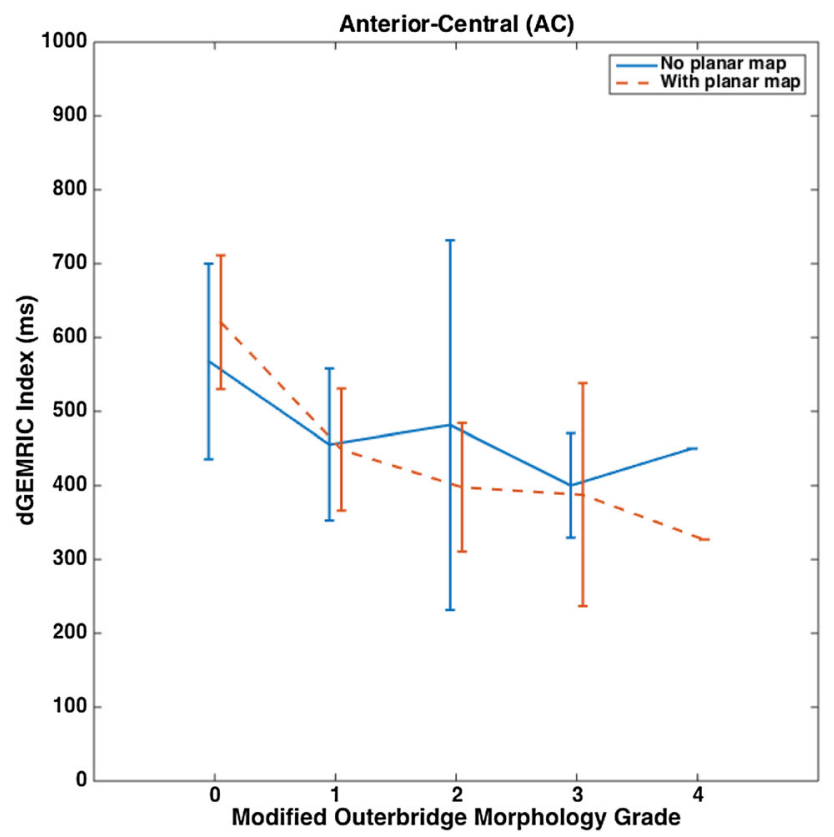

Fig. 4 A plot of the dGEMRIC index versus the modified Outerbridge grade for the AC subregion is shown with planar mapdependent and map-independent assessments plotted side by side. The mean values are connected by either solid or dashed lines and the error bars indicate \pm SD.

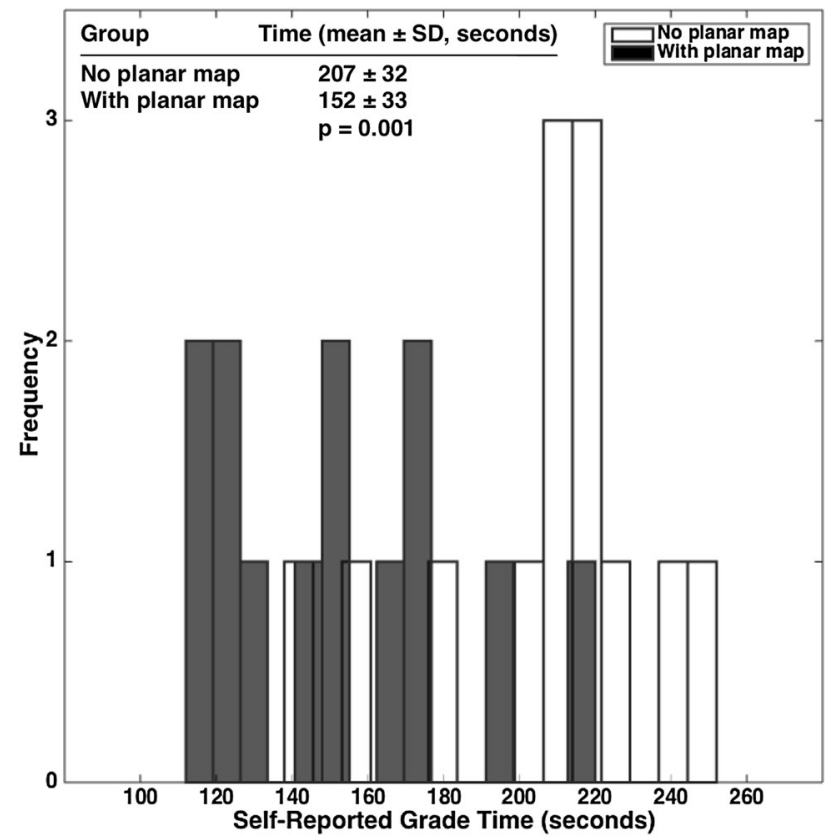

Fig. 5 A plot of radiological evaluation times comparing planar dGEMRIC map-dependent and map-independent assessment is shown. The Wilcoxon rank-sum test was used for the nonparametric test of significant difference between the two groups of radiological evaluation times.

(Tables 3,4). AP and SP are two subregions of acetabular cartilage that are most commonly damaged in hips with FAI [5], and damage to the corresponding central subregions would normally indicate poor surgical prognosis [6]. Therefore, our findings are promising for the potential of both standard MRI and dGEMRIC to help radiologists and surgeons predict the state of the joint that they would observe intraoperatively.

These results challenge the prior work by Bittersohl et al. [7], in which the authors reported a comparable correlation between standard MRI and intraoperative findings $(r=0.535, p<0.001)$ but a poor one between dGEMRIC and intraoperative findings $(\mathrm{r}=0.114, \mathrm{p}<$ 0.126) for hips with FAI. This difference in dGEMRIC findings may potentially be explained in two ways: (1) our analysis is specific to subregions of the acetabulum, whereas theirs pooled all examined subregions into one global comparison; and (2) we had 44 hips with both radiological and intraoperative findings, whereas they had only 16 hips, leading to weaker statistical power.

Meanwhile, our results with applying the planar dGEMRIC maps suggest that they do not notably alter the correlation between imaging-based and intraoperative assessments of acetabular cartilage damage in hips with symptomatic FAI. Although we did see a planar map-dependent change in the correlation between the dGEMRIC indices and intraoperative findings in $\mathrm{PC}\left(\mathrm{p}_{\text {diff }}=0.04\right.$; Table 4), the correlations in that subregion were quite small $(-0.2<\rho<0.2)$. It is worth mentioning that, although no map-dependent improvement is captured through assessment with radial reformats, it is possible that the planar maps alone may be sufficient to permit an accurate and comprehensive assessment of cartilage damage. Alternatively, the planar map may offer the greatest opportunity for improvement in MRI-based cartilage grading among less experienced readers, thereby reducing the impact of reader experience on the accuracy of the results. These hypotheses could be tested in future studies with radiologists and orthopaedic surgeons of different levels of experience.

Planar dGEMRIC maps may improve the correlation between the MRI grading of acetabular cartilage damage and the dGEMRIC index in FAI, but in a subregionspecific manner. We noted an improvement in this correlation strictly for the AC subregion $\left(\mathrm{p}_{\text {diff }}<0.001\right)$. Because damage to the central acetabular subregions has been suggested to be a poor surgical prognosticator for FAI [6], it is reassuring that referencing the planar maps significantly improves the correlation between MRI-based morphology and the dGEMRIC index in AC. This finding hints at a map-dependent improvement in the consistency between these two different modes of assessment. As far as we are aware, this is the first study to formally report such a correlation, and we believe it to have potential use in further guiding the analysis of imaging-based assessment of cartilage damage. 
Finally, the planar dGEMRIC map reduced the time that it took to perform Outerbridge grading and measure the dGEMRIC indices (Fig. 5). On average, evaluation times decreased by 55 seconds, or $27 \%$. Although this is arguably a minor change in the scheme of the entire radiological workflow, it does strongly suggest that a radiologist can use the map to obtain information about global cartilage biochemistry without having to invest additional time. The planar map is therefore potentially a costless workflow intervention. As researchers and developers evaluate future technologies, we encourage them to similarly bear the clinician's time in mind.

The fact that we found a moderate-to-strong, subregion-specific correlation between imaging-based and intraoperative assessments of acetabular cartilage damage in hips with symptomatic FAI suggests that dGERMIC at 1.5-T resolution can be reliably applied to study this patient population. Moreover, although using planar dGERMIC maps to guide standard evaluation does not appear to alter the correlation of imaging-based and intraoperative damage findings, it does improve the correlation between MRI-based morphology grading and biochemical signal specifically in the AC subregion. Importantly, the maps can offer radiologists more information about cartilage quality without having them incur significant time delay or lose evaluation accuracy. In the future, we would be interested in seeing whether the planar maps alone may be sufficient for accurate imagingbased assessment of cartilage damage. There is also potential benefit to conducting a survey of whether the planar maps are useful to hip orthopaedic surgeons as well as carrying out a more formal study of whether the maps significantly improve the surgeons' predictions of Beck's grades. Finally, we are interested in standardizing the signal that the planar maps display to permit comparison across hip records and also making the planar maps more quantifiable.

Acknowledgments We thank the Boston Children's Hospital's Departments of Orthopedic Surgery and Radiology, the Computational Radiology Laboratory, and Harvard Medical School for recordkeeping, consulting, and computing support. A special thanks is extended to Michelle Heinz for research support and Patricia Miller for statistical consultation.

\section{References}

1. Agricola R, Waarsing JH, Thomas GE, Carr AJ, Reijman M, Bierma-Zeinstra SM, Glyn-Jones S, Weinans H, Arden NK. Cam impingement: defining the presence of a cam deformity by the alpha angle: data from the CHECK cohort and Chingford cohort. Osteoarthritis Cartilage. 2014;22:218-225.
2. Anderson LA, Peters CL, Park BB, Stoddard GJ, Erickson JA, Crim JR. Acetabular cartilage delamination in femoroacetabular impingement: risk factors and magnetic resonance imaging diagnosis. J Bone Joint Surg Am. 2009;91:305-313.

3. Barer M. Role of innominate osteotomy in the treatment of children with Legg-Perthes disease. Clin Orthop Relat Res. 1978;135:2-9.

4. Bashir A, Gray ML, Hartke J, Burstein D. Nondestructive imaging of human cartilage glycosaminoglycan concentration by MRI. Magn Reson Med. 1999;41:857-865.

5. Beck M, Kalhor M, Leunig MGanz R. Hip morphology influences the pattern of damage to the acetabular cartilage: femoroacetabular impingement as a cause of early osteoarthritis of the hip. J Bone Joint Surg Br. 2005;87:1012-1018.

6. Beck M, Leunig M, Parvizi J, Boutier V, Wyss D, Ganz R. Anterior femoroacetabular impingement: part II. Midterm results of surgical treatment. Clin Orthop Relat Res. 2004;418:67-73.

7. Bittersohl B, Hosalkar HS, Apprich S, Werlen SA, Siebenrock KA, Mamisch TC. Comparison of pre-operative dGEMRIC imaging with intra-operative findings in femoroacetabular impingement: preliminary findings. Skeletal Radiol. 2011;40:553-561.

8. Cunningham T, Jessel R, Zurakowski D, Millis MB, Kim YJ. Delayed gadolinium-enhanced magnetic resonance imaging of cartilage to predict early failure of Bernese periacetabular osteotomy for hip dysplasia. J Bone Joint Surg Am. 2006;88:1540-1548.

9. Domayer SE, Mamisch TC, Kress I, Chan J, Kim YJ. Radial dGEMRIC in developmental dysplasia of the hip and in femoroacetabular impingement: preliminary results. Osteoarthritis Cartilage. 2010;18:1421-1428.

10. Felson D. Risk factors for osteoarthritis: understanding joint vulnerability. Clin Orthop Relat Res. 2004;427(Suppl):S16-21.

11. Jessel RH, Zilkens C, Tiderius C, Dudda M, Mamisch TC, Kim YJ. Assessment of osteoarthritis in hips with femoroacetabular impingement using delayed gadolinium enhanced MRI of cartilage. J Magn Reson Imaging. 2009;30:1110-1115.

12. Kim SD, Jessel R, Zurakowski D, Millis MB, Kim YJ. Anterior delayed gadolinium-enhanced MRI of cartilage values predict joint failure after periacetabular osteotomy. Clin Orthop Relat Res. 2012;470:3332-3341.

13. Kim YJ, Jaramillo D, Millis MB, Gray ML, Burstein D. Assessment of early osteoarthritis in hip dysplasia with delayed gadolinium-enhanced magnetic resonance imaging of cartilage. $J$ Bone Joint Surg Am. 2003;85:1987-1992.

14. Lattanzi R, Petchprapa C, Glaser C, Dunham K, Mikheev AV, Krigel A, Mamisch TC, Kim YJ, Rusinek H, Recht M. A new method to analyze dGEMRIC measurements in femoroacetabular impingement: preliminary validation against arthroscopic findings. Osteoarthritis Cartilage. 2012;10:1127-1133.

15. March L, Smith EU, Hoy DG, Cross MJ, Sanchez-Riera L, Blyth F, Buchbinder R, Vos T, Woolf AD. Burden of disability due to musculoskeletal (MSK) disorders. Best Pract Res Clin Rheumatol. 2014;28:353-366.

16. Meng XL, Rosenthal R, Rubin D. Comparing correlated correlation coefficients. Psychol Bull. 1992;111:172-175.

17. Nepple JJ, Larson CM, Smith MV, Kim YJ, Zaltz I, Sierra RJ, Clohisy JC. The reliability of arthroscopic classification of acetabular rim labrochondral disease. Am J Sports Med. 2012; 40:2224-2229.

18. R Project for Statistical Computing. Available at: http://www.rproject.org/. Accessed June 9, 2015.

19. Siversson C, Akhondi-Asl A, Bixby S, Kim YJ, Warfield SK. Three-dimensional hip cartilage quality assessment of morphology and dGEMRIC by planar maps and automated segmentation. Osteoarthritis Cartilage. 2014;22:1511-1515. 
20. Steiger J. Tests for comparing elements of a correlation matrix. Psychol Bull. 1980;87:245-251.

21. Venn M, Maroudas A. Chemical composition and swelling of normal and osteoarthritic femoral head cartilage. Ann Rheum Dis. 1977;36:399-406.
22. Yushkevich PA, Piven J, Hazlett HC, Smith RG, Ho S, Gee JC, Gerig G. User-guided 3D active contour segmentation of anatomical structures: significantly improved efficiency and reliability. Neuroimage. 2006;31:1116-1128. 